\title{
Edge-emitting polariton laser and amplifier based on a $\mathrm{ZnO}$ waveguide
}

\author{
O. Jamadi ${ }^{1}$, F. Reveret ${ }^{1}$, P. Disseix', F. Medard', J. Leymarie', A. Moreau', D. Solnyshkov', C. Deparis², M. Leroux², \\ E. Cambril' ${ }^{3}$ S. Bouchoule ${ }^{3}$, J. Zuniga-Perez ${ }^{2}$ and G. Malpuech ${ }^{1}$
}

\begin{abstract}
We demonstrate edge-emitting exciton-polariton (polariton) laser operation from 5 to $300 \mathrm{~K}$ and polariton amplifiers based on polariton modes within $\mathrm{ZnO}$ waveguides. The guided mode dispersion below and above the lasing threshold is directly measured using gratings placed on top of the sample, fully demonstrating the polaritonic nature of the lasing modes. The threshold is found to be smaller than that expected for radiative polaritons in planar $\mathrm{ZnO}$ microcavities below $150 \mathrm{~K}$ and comparable above. These results open up broad perspectives for guided polaritonics by enabling easier and more straightforward implementation of polariton integrated circuits that exploit fast propagating polaritons, and, possibly, topological protection.
\end{abstract}

\section{Introduction}

Exciton-polaritons (polaritons) are quasi-particles that result from the coupling between a light mode and an excitonic resonance. Polaritons were theoretically introduced by Hopfield ${ }^{1}$ and Agranovich ${ }^{2}$ at the end of the $50^{\prime} \mathrm{s}$ to describe light propagation in bulk semiconductors. In 1992, the achievement of strong lightmatter coupling between the photonic radiative modes of a planar microcavity and the excitonic resonances of embedded quantum wells ${ }^{3}$ opened up the era of twodimensional cavity polaritons, which have since been extensively studied. From the fundamental side, polaritons represent a direct implementation of a spinor quantum fluid of light ${ }^{4,5}$, with unique access to the timedependent wavefunction in real and reciprocal space via optical measurements, which enables study of BoseEinstein condensation in open systems ${ }^{6-8}$, of the superfluidity of light ${ }^{9,10}$, and of various types of topological defects $^{11,12}$. In-plane potentials have been commonly realized for a decade. These potentials enable one to

\footnotetext{
Correspondence: G Malpuech (guillaume.malpuech@uca.fr)

${ }^{1}$ Institut Pascal, PHOTON-N2, Université Clermont Auvergne, CNRS, SIGMA

Clermont, F-63000 Clermont-Ferrand, France

${ }^{2}$ UCA, CRHEA-CNRS, Valbonne F-06560, France

Full list of author information is available at the end of the article.
}

implement coupled $0 \mathrm{D}$ polariton modes, building artificial molecules $^{13}$ or lattices ${ }^{14-17}$, which opens up new perspectives for emulating different physical systems, such as topological insulators ${ }^{18}$, topological polariton lasers ${ }^{19,20}$, or the classical XY model ${ }^{21}$. From the applied side, polaritons have the critical advantage of having a high nonlinear response, low-threshold operation, and potentially high scalability ${ }^{10}$ and are useful for realizing lowconsumption, compact, all-optical devices, which could replace electronics for some tasks.

The most paradigmatic polariton device is the so-called polariton laser ${ }^{22}$, based on polariton condensation, which does not require electron-hole gain and can therefore exhibit a very low threshold. In GaAs-based samples, at $5 \mathrm{~K}$, a wide variety of devices, such as switches and optical transistors, have been demonstrated (for a review see ref. ${ }^{10}$ ). Most of these devices are based on the creation of a polariton flow based on slow radiative modes propagating at $1-2 \%$ of the speed of light, which is only possible at low temperature in very high $Q$ samples (typically $\left.Q \sim 10^{5}\right)$. Room-temperature polaritonics requires the use of microcavities based on large-bandgap semiconductors $(\mathrm{GaN}, \mathrm{ZnO})$ or various organic materials, where polariton lasing has also been demonstrated ${ }^{23-28}$. However, their Qfactors remain limited to a few thousand, which makes it

\section{(c) The Author(s) 2018}

(c) (i) Open Access This article is licensed under a Creative Commons Attribution 4.0 International License, which permits use, sharing, adaptation, distribution and reproduction cc) in any medium or format, as long as you give appropriate credit to the original author(s) and the source, provide a link to the Creative Commons license, and indicate if changes were made. The images or other third party material in this article are included in the article's Creative Commons license, unless indicated otherwise in a credit line to the material. If material is not included in the article's Creative Commons license and your intended use is not permitted by statutory regulation or exceeds the permitted use, you will need to obtain permission directly from the copyright holder. To view a copy of this license, visit http://creativecommons.org/licenses/by/4.0/. 


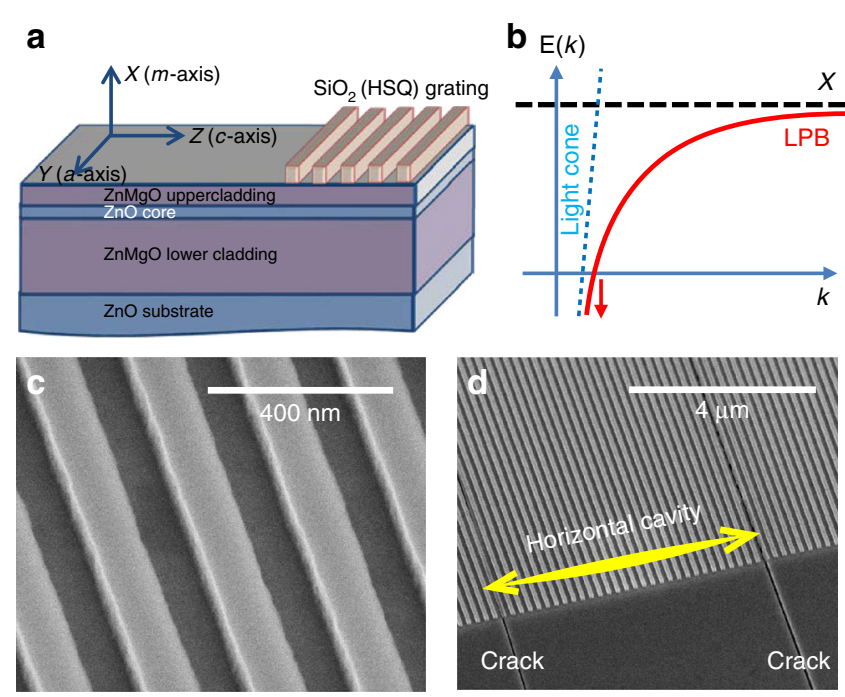

Fig. 1 Description of sample W1. a Scheme for the sample. The guided mode propagates along the Z-axis in the ZnO core. $\mathbf{b}$ Sketch of the lower branch (LPB) of an exciton-polariton guided mode. The arrows symbolize the absence of a finite energy minimum. c Scanning electron microscope (SEM) image of the $\mathrm{SiO}_{2}$ grating deposited on top of the waveguide. $\mathbf{d}$ SEM image of the sample showing two typical horizontal cracks here separated by $18 \mu \mathrm{m}$, nearly parallel to the grating

difficult to implement room-temperature polariton-based switches that exploit concepts developed in GaAs-based samples.

An alternative geometry for polaritonics is one where either Bloch surface waves ${ }^{29-31}$, or guided modes are confined by total internal reflection in a layer ${ }^{32-38}$, strongly coupled to excitonic resonances. The geometry is very appealing because of its simple technological realization, easier electrical injection, and the possibility it opens up to design integrated polaritonic circuits with very limited radiative losses. Guided polaritons have been observed and studied in $\mathrm{GaAs}^{33,34}$, organic materials ${ }^{30,35}$, $\mathrm{GaN}^{36}$, and transitional metal dichalcogenides ${ }^{37}$ (TMD), with recent reports of nonlinear polariton-polariton interaction $^{31,38}$. This horizontal geometry is very favorable for straightforward implementation of polariton integrated circuits with the use of topological protection, such as in the recently reported "topological lasers" 39,40 . However, even if theoretically predicted ${ }^{32}$, "horizontal" edge-emitting polariton lasing in such fast-propagating polariton modes has never been observed.

In this work, we realize one of the crucial milestones of polaritonics by reporting lasing in $\mathrm{ZnO}$-based waveguides from 5 to $300 \mathrm{~K}$ and the amplification of guided polariton modes under optical pumping. The mode dispersion below and above the lasing threshold is directly measured using gratings placed on the top of the sample. These dispersions clearly demonstrate the polaritonic nature of the lasing modes.

\section{Results}

We use two $\mathrm{ZnO}-\mathrm{ZnMgO}$ waveguides grown by molecular beam epitaxy onto $\mathrm{m}$-plane bulk $\mathrm{ZnO}$ substrates. The thicknesses of the active $\mathrm{ZnO}$ layers are $50 \mathrm{~nm}$ (W1 sample) and $130 \mathrm{~nm}$ (W2 sample), respectively. A sketch of the sample W1 is shown in Fig. 1a, whereas a sketch of the guided exciton-polariton dispersion (lower branch) is shown in Fig. 1b. At low energies, the polariton dispersion is photon-like: linear in wave vector and showing no energy minimum, which is a key difference with respect to $2 \mathrm{D}$ cavity polaritons. W1 is covered by sets of $\mathrm{SiO}_{2}$ gratings (Fig. 1c), perpendicular to the $\mathrm{ZnO} c$-axis, with period $\Lambda$. The shift of the propagation constant by $2 \pi / \Lambda$ allows direct access to the polariton guided mode dispersion. The sample W2 is a half-microcavity without any grating. The sample geometries together with fabrication details are presented in Supplementary material and in ref. ${ }^{41}$. The common aspect of both samples is the presence of regular horizontal cracks, as one can see in the SEM image (Fig. 1d). These cracks appear because of the mismatch of the lattice parameter and the thermal expansion coefficient between $\mathrm{ZnO}$ and $\mathrm{ZnMgO}$. They occur perpendicular to the $c$-axis of the crystal and are typically separated by $5-40 \mu \mathrm{m}$. By reflecting light they induce a substantial confinement leading to the formation of a genuine horizontal cavity for the guided modes. Their presence plays a crucial role in the success of our observations compared with the previous experimental studies of polariton waveguides. 
The micro-photoluminescence of the samples is studied by quasi-cw excitation using 400-ps-long pulses (Methods section), much longer than all relevant time scales in the system, which enables the establishment of a steady state regime. The power dependencies of the emission of W1 at 5 and $300 \mathrm{~K}$ are shown in Fig. 2a, b, demonstrating a very clear nonlinear threshold at $P_{\text {th }}$. The horizontal cavity sizes are $23 \mu \mathrm{m}$ and $27 \mu \mathrm{m}$ at $5 \mathrm{~K}$ and $300 \mathrm{~K}$, respectively. They are entirely covered by the pump spot and we do not use spatial selection of the emission, which originates from both the grating and the cracks. The guided polariton mode dispersions at 5 and $300 \mathrm{~K}$, below and above threshold, are shown in Figs. 2c-f, respectively. The optimal grating periods are $\Lambda=190 \mathrm{~nm}$ at $5 \mathrm{~K}$ and $\Lambda=$ $180 \mathrm{~nm}$ at $300 \mathrm{~K}$. All measured dispersions clearly deviate from the bare photonic mode (shown by the dashed line together with the bare A-exciton energy). At $5 \mathrm{~K}$, the main emission below threshold arises from donor-bound excitons $\left(\mathrm{D}^{0} \mathrm{X}\right.$ ) located $\sim 15 \mathrm{meV}$ below the A-exciton energy. The emission from the cracks appears as a weak flat emission line, which can be removed by spatially selecting the emission from the grating only (Supplementary Fig. S2). The measured polariton dispersion is well reproduced by a two-oscillator model where both $A$ and $B$ excitonic resonances are modeled as a single oscillator placed at the A-exciton energy. We take a linear photon dispersion with a slope fixed by that of the measured dispersions at $\sim 3.1 \mathrm{eV}$. The extracted Rabi splitting is $\Omega_{\mathrm{R}}$ $=224 \mathrm{meV}$ at low pumping for both temperatures. At $P_{\mathrm{th}}$, the dispersion remains essentially unchanged at $5 \mathrm{~K}\left(\Omega_{\mathrm{R}}\right.$ $=222 \mathrm{meV}$ ). Polariton lasing takes place in modes having an exciton fraction of $\sim 90 \%$. A movie with emission images vs. angle and energy over a wide pumping range $\left(0.1-20 P_{\text {th }}\right)$ is presented in the Supplementary material. An increase in the pumping intensity induces a blueshift for the modes, as shown in Fig. 2a, b and Supplementary Fig. S3, due to screening of the exciton oscillator strength. This screening effect is more significant at $300 \mathrm{~K}$ because of the larger pumping threshold value, with the fit giving $\Omega_{\mathrm{R}}=180 \mathrm{meV}$, which is $20 \%$ weaker than at low pumping (Fig. 2f). Polariton lasing occurs in more photon-like states than at $5 \mathrm{~K}$, as theoretically expected ${ }^{32}$, but still with a substantial $50-60 \%$ excitonic fraction. The velocity of these modes is $\sim 40-50 \%$ of that of the bare photonic mode of the waveguide, $\sim 60 \mu \mathrm{m} / \mathrm{ps}$. In general, an increase of the pumping density leads to a blueshift of the dispersion due to the screening effect as well as to a faster polariton relaxation along this dispersion. In 2D cavities, polaritons cannot relax energetically below their ground state, and the blueshift of the dispersion leads to a blueshift of the emission. With guided polaritons, there is no energy minimum, and, thus, a faster energy relaxation along the dispersion leads to a redshift of the emission, despite the fact that the dispersion as a whole is blueshifted. This redshift, clearly visible in the movie and in Supplementary Fig. S4 and S5, and well reproduced by simulations based on semi-classical Boltzmann equations (Supplementary Fig. S6), is due to the faster polariton relaxation rates at higher densities.

Interestingly, the lasing mechanism in $\mathrm{ZnO}$-based systems, such as nanowires ${ }^{42}$, has been a matter of debate for quite a long time. Polariton lasing ${ }^{43}$ was considered as a possible mechanism for over a decade ${ }^{44-46}$, but the absence of direct dispersion measurements made it difficult to draw a definite conclusion. The direct dispersion measurements we report enable one to clearly establish the polaritonic origin for the emission above the nonlinear threshold. The temperature dependences between 5 and $300 \mathrm{~K}$ for the polariton lasing energy and of the A-exciton energy are shown in Fig. 2g. The difference between the two energies increases slightly, which demonstrates that lasing occurs in more and more photonic polariton states vs. temperature. This behavior is expected from a previous numerical study of this system ${ }^{32}$ and from previous works performed on planar cavities ${ }^{7,47,26,48}$. Indeed, similar to the increase in pumping, the increase of temperature leads to faster relaxation along the polariton branch, which enables lasing to take place at lower polariton states.

Figure $2 \mathrm{~h}$ shows a comparison of the thresholds measured in W1 (horizontal polariton lasing) and those of a vertical polariton laser measured in a full microcavity displaying a quality factor of 2000 (ref. ${ }^{47}$ ), which corresponds to a cavity photon lifetime of $0.4 \mathrm{ps}$. Thresholds are comparable, and they are even slightly lower for the horizontal polariton laser in a wide temperature range, which can be understood qualitatively as follows. In both cases, the main polariton scattering mechanism was termed "excitonic gain" in the $60 \mathrm{~s}$ (ref. ${ }^{49}$ ), which involves the scattering of an exciton-like polariton with either another exciton-like polariton or with a LO-phonon towards a polariton state with lower energy and thus with a larger photonic fraction. In vertical cavities, polariton lasing takes place if excitonic gain is efficient enough compared with the polariton lifetime. In a guided mode geometry, the scattering rate should be compared with the transit time of polaritons under the pumping spot. Considering a typical $20 \mu \mathrm{m}$ size pumping area and polariton guided modes propagating at $13 \mu \mathrm{m} / \mathrm{ps}$ at $5 \mathrm{~K}$ and $60 \mu \mathrm{m} /$ ps at $300 \mathrm{~K}$, the transit time of polaritons under the spot is $1.5 \mathrm{ps}$ and $0.33 \mathrm{ps}$, respectively (neglecting the feedback provided by the horizontal cavities), comparable with the 0.4 ps of a vertical $\mathrm{ZnO}$-based cavity ${ }^{47}$. This simple estimate explains the similarity of the measured thresholds in the two types of samples, and even the lower threshold of the horizontal polariton laser at $5 \mathrm{~K}$. This estimate also suggests that in thick microcavities, which support both radiative and guided modes, the latter are responsible for 

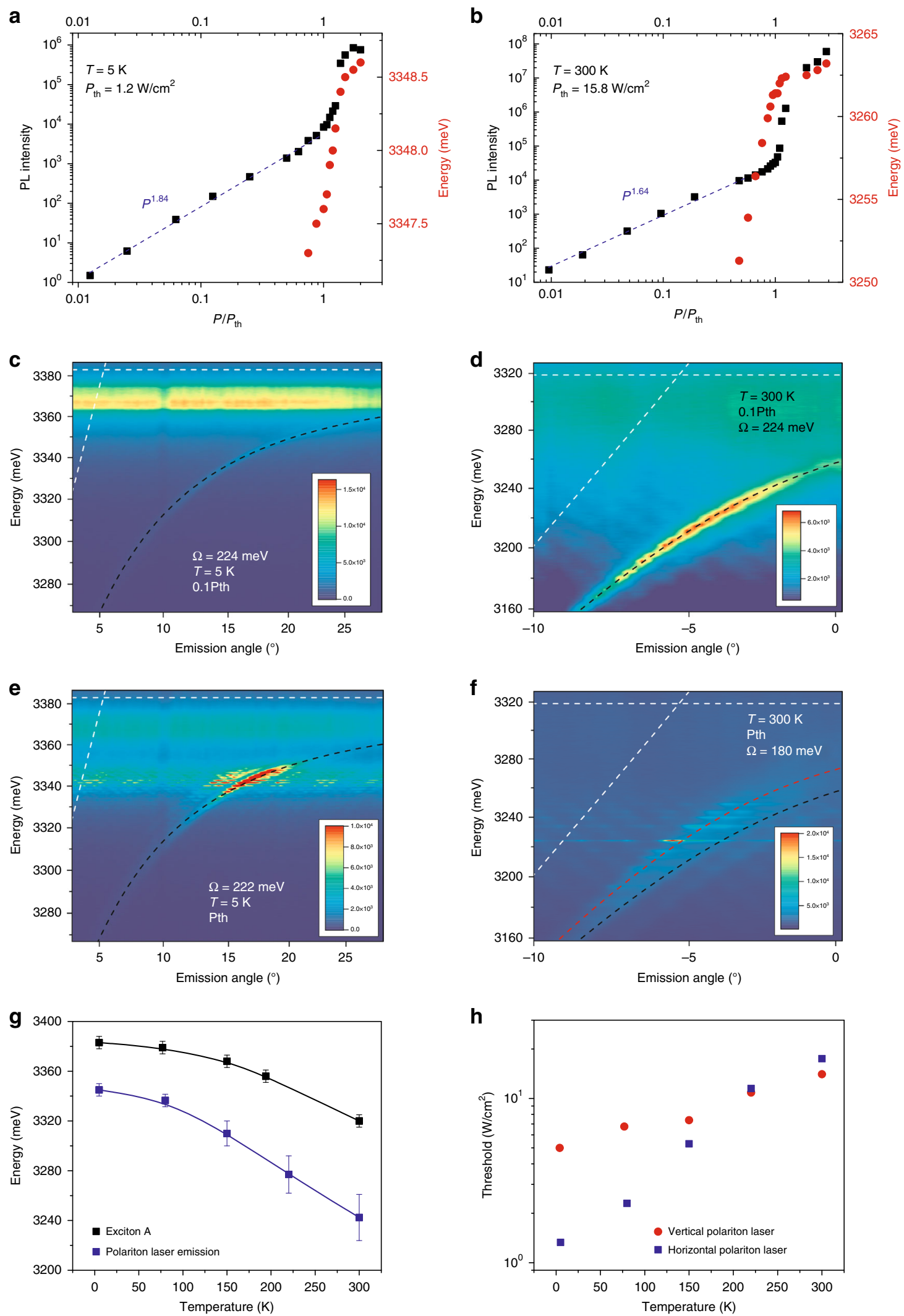

Fig. 2 (See legend on next page.) 
(see figure on previous page)

Fig. $\mathbf{2}$ Emission of $\mathbf{W} \mathbf{1}$ at $\mathbf{5}$ and $\mathbf{3 0 0} \mathbf{K}$. The pump spot covers the entire horizontal cavity length. a $5 \mathrm{~K}$. Power dependence of the emission (black squares) and energy at the wave vector of the most intense lasing peaks. b Same as a, but at $300 \mathrm{~K}$. c-f Energy of emission vs. angle (dispersion). The white dashed lines are the expected bare TEO photon and exciton modes. The black dashed lines are the strongly coupled polariton modes with the $\Omega_{\mathrm{R}}$ indicated in each panel. The intensities are given in arbitrary units. c $5 \mathrm{~K}$ and $0.1 P_{\mathrm{th}}, \mathbf{d} 300 \mathrm{~K}$ and $0.1 P_{\mathrm{th}}, \mathbf{e} 5 \mathrm{~K}$ and $P_{\text {th }}$, f $300 \mathrm{~K}$ and $P_{\text {th }}$. The red dashed lines in $\mathbf{f}$ is the polariton dispersion with $\Omega_{R}=224 \mathrm{meV}$. $\mathbf{g}$ Experimental average exciton energies plotted together with the average horizontal polariton laser emission energies vs. temperature. The solid black line corresponds to Viña's law and the blue line is a guide for the eye. h Polariton lasing threshold vs. temperature for a horizontal polariton laser in W1 (blue squares) and for a vertical cavity polariton laser studied in ref. ${ }^{48}$ (red stars)

a

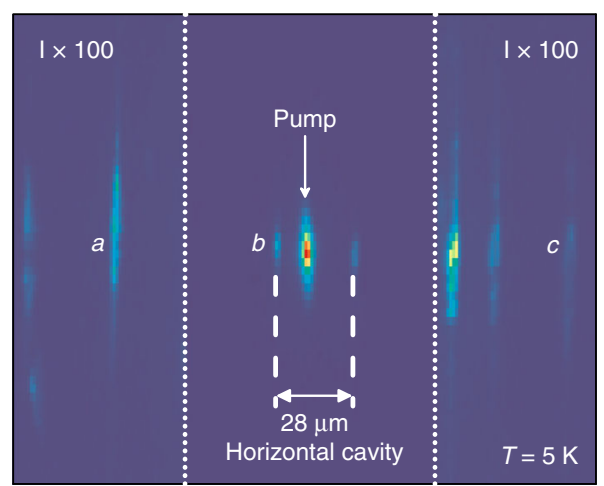

C

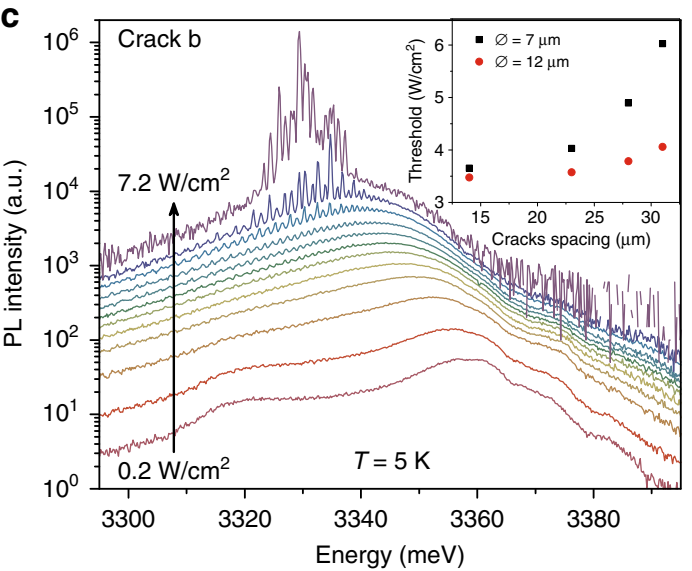

b

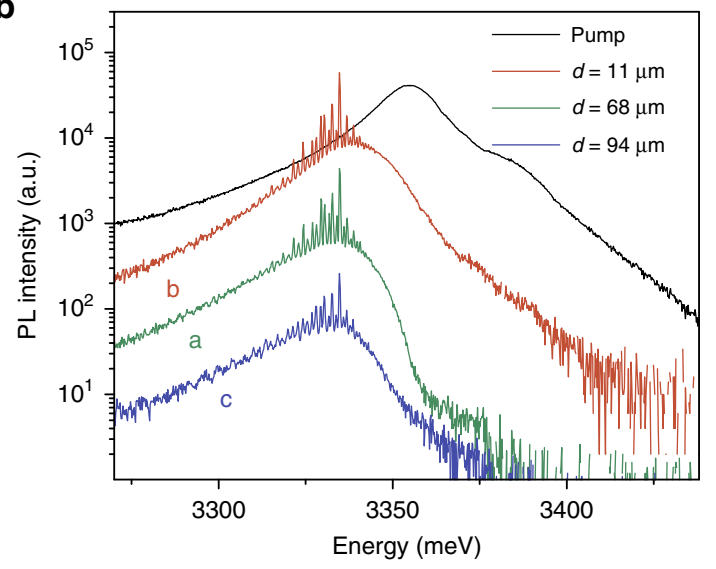

d

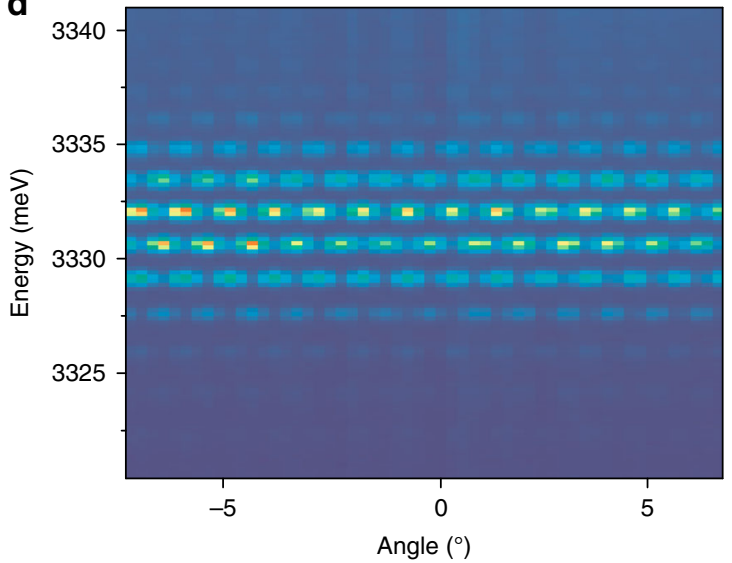

Fig. 3 Sample W2. a Real space emission at 5K. The intensity (I) of the two lateral regions is magnified by 100 . $\mathbf{b}$ Emission spectra from the pump

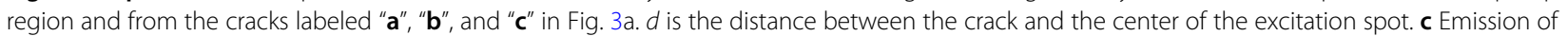
the crack "b" vs. pumping power, showing the emergence of the Fabry-Perot modes of the horizontal cavity together with the onset of lasing (power dependence is shown in Supplemental Material). The inset shows the threshold values vs. the cavity size for two different pump diameters. $\mathbf{d}$ Far-field image zoomed in onto the fringes resulting from the interference of the emission from two distant cracks, demonstrating their mutual coherence

strong losses affecting vertical polariton lasing. In a cracked homoepitaxial full cavity (completed with a top dielectric DBR), we have been able to observe lasing simultaneously in the vertical radiative modes and in the horizontal guided modes (not shown).

Another interesting feature is the emergence of sharp emission lines, appearing at threshold within the polariton dispersion. These lines are the Fabry-Perot modes of the horizontal cavity formed by the two cracks surrounding the pump. The rise of these sharp peaks demonstrates the onset of phase coherence for the polariton modes all along the horizontal cavity, as explained below. Figure 3a shows the real space emission at $5 \mathrm{~K}$ of the sample W2 excited above $P_{\text {th }}$ by a 7- $\mu$ m-sized pump (four times smaller than the distance between the surrounding cracks). The emission spectra from the pump area and from the three different cracks are shown in Fig. 3b. The pump area shows the radiative $\mathrm{ZnO}$ emission dominated 

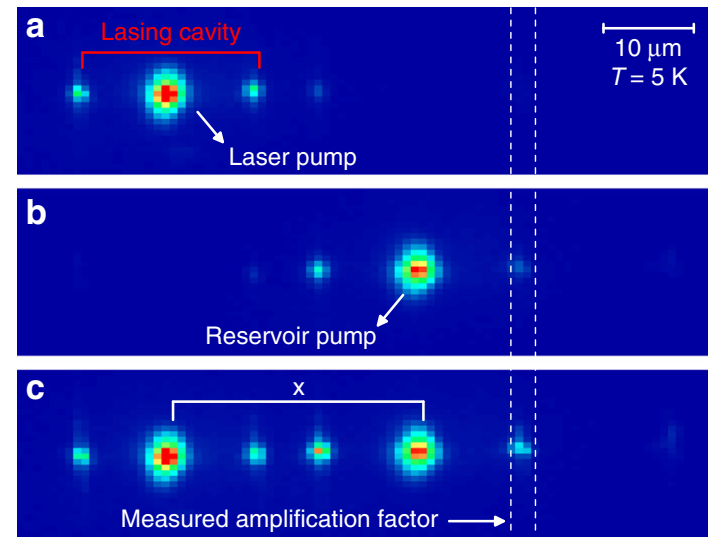

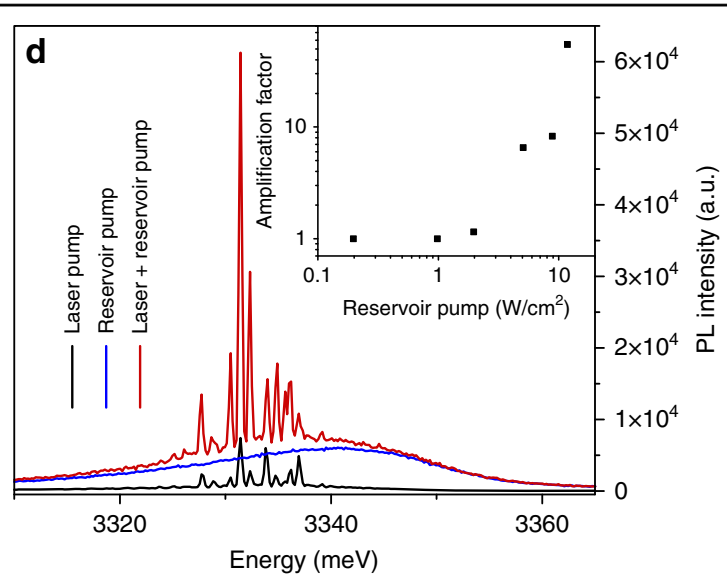

Energy (meV)

Fig. 4 Horizontal polariton amplifier regime at $\mathbf{5 K}$ in W2. $\mathbf{a}$, b, c Real space emission with: a only "laser pump" (above lasing threshold); $\mathbf{b}$ only "reservoir pump" (below lasing threshold); c both pump spots ("laser" and "reservoir"). The vertical dashed lines show the crack where the amplification is measured. $\mathbf{d}$ Emission spectra extracted through the crack at the right of the reservoir pump ( $60 \mu \mathrm{um}$ from the laser pump). The "reservoir" pump power is $8.9 \mathrm{~W} / \mathrm{cm}^{2}$, which is below threshold for this specific cavity as one can see from the emission spectrum when only the "reservoir pump" is ON. The "laser" pump power is $6.9 \mathrm{~W} / \mathrm{cm}^{2}$-above threshold for this cavity. The amplification factor is defined as the ratio between the most intense lasing peaks. The inset shows its evolution as a function of the reservoir pump

by the first Bragg mode of the mirror at $3355 \mathrm{meV}$ and the $\mathrm{D}^{0} \mathrm{X}$ lines at $3370 \mathrm{meV}$. The free exciton line is visible as a shoulder at $\sim 3385 \mathrm{meV}$. The emission from the cracks shows the Fabry-Perot interference peaks. The powerdependent emission spectra measured at crack "b", demonstrating the emergence of the horizontal FabryPerot modes above threshold, are shown in Fig. 3c. The interference fringes are weakly visible in the pump area since there is no well-defined out-coupling mechanism for the guided modes.

Figure $3 \mathrm{~d}$ is a far-field image (taken from a region without the grating) showing the emission from two distant cracks. The resulting interference pattern shows that at a given energy there is no significant phase fluctuation in the emission from one crack with respect to the other. The mutual coherence extracted from the fringe contrast can reach values close to $70 \%$. The inset of Fig. 3c shows the influence of the spot size and of the distance between cracks on $P_{\text {th }}$. As one would expect, the smallest threshold is achieved when the pump and cavity size are comparable. However, the dependence is relatively weak, with a pump approximately four times smaller than the cavity showing only a doubled threshold, because the residual absorption in the region without pumping is extremely reduced at the polariton energy. This weak dependence is typical for the polariton lasing mechanism, which enables gain (polariton stimulated scattering) to take place well below the energy of electronic transitions.

The detailed analysis of the Fabry-Perot modes is performed in section IV of supplementary material. The fringe spacing is compatible with the crack spacing, but more than that, the change of the inter-fringe with energy for a known crack separation enables indirect extraction of the dispersion. This method is commonly used in the study of nanowires ${ }^{44,45}$, where direct dispersion measurements are difficult. The result of extraction is shown in Supplementary Fig. S7 and reveals a curved dispersion typical for polaritons with $\Omega_{\mathrm{R}} \sim 200 \mathrm{meV}$.

Next, we use a two-spot experiment to demonstrate how the horizontal polariton laser emission created in a given cavity can propagate and be re-amplified several tens of microns away by another pump, being itself below the lasing threshold (Fig. 4). A first spot, named "laser pump", excites a horizontal cavity, creating the laser signal to be amplified (Fig. 4a). A second spot, named "reservoir pump", is placed in another horizontal cavity, at a distance of $60 \mu \mathrm{m}$ from the laser pump (Fig. 4b). The power density of the laser pump is fixed above threshold, while the power density of the reservoir pump is kept below threshold. The emission is collected at the crack nearest to the reservoir pump, which shows low emission intensity when only one of the two pumps is ON. Figure 4c shows the configuration when both spots are turned ON. The emission spectra from the selected crack are displayed for each of the three previous pumping schemes in Fig. $4 \mathrm{~d}$. The horizontal polariton laser signal is amplified when the two pumps are ON. The amplification factor is calculated as the ratio between the emission maxima measured when both pumps are $\mathrm{ON}$ and when only the laser pump is ON. The highest amplification factor is 55 (inset of Fig. 4d), which is reached when the reservoir pump is just below its lasing threshold, demonstrating that the signal created by the laser pump, which can be easily identified by its free-spectral range defined by the initial cavity and which decays by approximately one order of magnitude when reaching the reservoir pump, is 
strongly re-amplified. This scheme can also be interpreted as an optical transistor, with the transmission of a signal from a source (the laser pump) to a drain (the crack) being modulated by a control gate (the reservoir).

\section{Discussion}

We demonstrated a horizontal polariton laser and amplifier that are much simpler to fabricate and process than full vertical microcavities and are simpler to manipulate than nanowires with which our system shares many similarities. One crucial step for polaritonics is electrical injection ${ }^{25,50}$, which is strongly facilitated in this horizontal geometry. Thus, the whole family of model devices implemented at low temperature in GaAs-based samples can probably be implemented at room temperature under electrical injection using alternative materials ( $\mathrm{ZnO}, \mathrm{GaN}$, organics, TMD, etc.), while displaying greatly reduced photonic losses due to the guided modes. It should be noted that polariton topological insulators with protected edge states have recently been proposed ${ }^{18}$. Topological polariton lasers have been observed in 1D systems, and topological lasers based on topological inplane cavities have been realized ${ }^{39,40}$. Topological inplane arbitrary cavities could probably be implemented for polaritons, which when combined with the present observation could enable realization of $2 \mathrm{D}$ topological polariton lasers with a low threshold and high beam quality that can operate at room temperature. These exciting perspectives open up the way to many studies in the future.

\section{Materials and methods}

The samples were studied through microphotoluminescence using the fourth harmonic $(266 \mathrm{~nm})$ of a Nd:YAG laser as the excitation source. The pulse duration was $400 \mathrm{ps}$ and the repetition rate was $20 \mathrm{kHz}$. To make a comparison with cw excitation, one, therefore, has to multiply the average quasi-cw pumping power we use by a factor of 125,000 . A UV microscope objective with a 0.4 numerical aperture was used to obtain small excitation spots with varying diameters. The emission of the sample was collected through the same objective and imaged onto the spectrometer slit by a spherical lens mounted on a motorized translation stage.

\footnotetext{
Acknowledgements

We acknowledge the support of the ANR projects: "Plug and Bose" (ANR-16CE24-0021), "Quantum Fluids of Light" (ANR-16-CE30-0021) and the "Investissements d'avenir" program GANEX (ANR-11-LABX-004), IMOBS3 (ANR10-LABX-16-01), ISITE "Cap2025" (16-IDEX-0001). C2N is a member of RENATECH (CNRS), the national network of large micro-nanofabrication facilities. S.B. thank Dr. F. Raineri of C2N for fruitful discussions. D.D.S. acknowledges the support of IUF (Institut Universitaire de France).

\section{Author details}

${ }^{1}$ Institut Pascal, PHOTON-N2, Université Clermont Auvergne, CNRS, SIGMA Clermont, F-63000 Clermont-Ferrand, France. ${ }^{2}$ UCA, CRHEA-CNRS, Valbonne
}

F-06560, France. ${ }^{3}$ Centre Nanosciences et Nanotechnologies (C2N), CNRS, University Paris-Saclay, Marcoussis F-91460, France

Conflict of interest

The authors declare that they have no conflict of interest.

Supplementary information is available for this paper at https://doi.org/ 10.1038/s41377-018-0084-z.

Received: 4 April 2018 Revised: 26 September 2018 Accepted: 26 September 2018

Published online: 31 October 2018

\section{References}

1. Hopfield, J. J. Theory of the contribution of excitons to the complex dielectric constant of crystals. Phys. Rev. 112, 1555-1567 (1958).

2. Agranovič, V. M. Dispersion of electromagnetic waves in crystals. Ž. Ėksper Teor. Fiz. 37, 307-441 (1960).

3. Weisbuch, C., Nishioka, M., Ishikawa, A. \& Arakawa, Y. Observation of the coupled exciton-photon mode splitting in a semiconductor quantum microcavity. Phys. Rev. Lett. 69, 3314-3317 (1992).

4. Shelykh, I. A., Kavokin, A. V., Rubo, Y. G., Liew, T. C. H. \& Malpuech, G. Polariton polarization-sensitive phenomena in planar semiconductor microcavities. Semicond. Sci. Technol. 25, 013001 (2010).

5. Carusotto, I. \& Ciuti, C. Quantum fluids of light. Rev. Mod. Phys. 85, 299-366 (2013).

6. Kasprzak, J. et al. Bose-einstein condensation of exciton polaritons. Nature $\mathbf{4 4 3}$ 409-414 (2006).

7. Kasprzak, J., Solnyshkov, D. D., André, R., Dang, L. S. \& Malpuech, G. Formation of an exciton polariton condensate: thermodynamic versus kinetic regimes. Phys. Rev. Lett. 101, 146404 (2008).

8. Sun, Y. B. et al. Bose-Einstein condensation of long-lifetime polaritons in thermal equilibrium. Phys. Rev. Lett. 118, 016602 (2017).

9. Amo, A. et al. Superfluidity of polaritons in semiconductor microcavities. Nat. Phys. 5, 805-810 (2009).

10. Sanvitto, D. \& Kéna-Cohen, S. The road towards polaritonic devices. Nat. Mater 15, 1061-1073 (2016).

11. Lagoudakis, K. G. et al. Quantized vortices in an exciton-polariton condensate. Nat. Phys. 4, 706-710 (2008).

12. Hivet, R. et al. Half-solitons in a polariton quantum fluid behave like magnetic monopoles. Nat. Phys. 8, 724-728 (2012).

13. Sala, V. G. et al. Spin-orbit coupling for photons and polaritons in microstructures. Phys. Rev. X 5, 011034 (2015).

14. Lai, C. W. et al. Coherent zero-state and $\pi$-state in an exciton-polariton condensate array. Nature 450, 529-532 (2007).

15. Jacqmin, $T$. et al. Direct observation of Dirac cones and a flatband in a honeycomb lattice for polaritons. Phys. Rev. Lett. 112, 116402 (2014).

16. Kim, N. Y. et al. f-band condensates in exciton-polariton lattice systems. Phys. Rev. B 89, 085306 (2014)

17. Whittaker, C. E. et al. Exciton polaritons in a two-dimensional lieb lattice with spin-orbit coupling. Phys. Rev. Lett. 120, 097401 (2018).

18. Nalitov, A. V., Solnyshkov, D. D. \& Malpuech, G. Polariton Z topological insulator. Phys. Rev. Lett. 114, 116401 (2015).

19. Solnyshkov, D. D., Nalitov, A. V. \& Malpuech, G. Kibble-zurek mechanism in topologically nontrivial zigzag chains of polariton micropillars. Phys. Rev. Lett. 116, 046402 (2016).

20. St-Jean, P. et al. Lasing in topological edge states of a one-dimensional lattice. Nat. Photonics 11, 651-656 (2017).

21. Berloff, N. G. et al. Realizing the classical XY Hamiltonian in polariton simulators. Nat. Mater. 16, 1120-1126 (2017).

22. Imamoğlu, A., Ram, R. J., Pau, S. \& Yamamoto, Y. Nonequilibrium condensates and lasers without inversion: exciton-polariton lasers. Phys. Rev. A. 53, 4250-4253 (1996).

23. Christopoulos, S. et al. Room-temperature polariton lasing in semiconductor microcavities. Phys. Rev. Lett. 98, 126405 (2007).

24. Christmann, G., Butté, R., Feltin, E., Carlin, J. F. \& Grandjean, N. Room temperature polariton lasing in a GaN-AlGaN multiple quantum well microcavity. Appl. Phys. Lett. 93, 51102 (2008). 
25. Bhattacharya, P. et al. Room temperature electrically injected polariton laser. Phys. Rev. Lett. 112, 236802 (2014).

26. Li, F. et al. From excitonic to photonic polariton condensate in a $\mathrm{ZnO}$-based microcavity. Phys. Rev. Lett. 110, 196406 (2013).

27. Kéna-Cohen, S. \& Forrest, S. R. Room-temperature polariton lasing in an organic single-crystal microcavity. Nat. Photonics 4, 371-375 (2010).

28. Dietrich, C. P. et al. An exciton-polariton laser based on biologically produced fluorescent protein. Sci. Adv. 2, e1600666 (2016)

29. Liscidini, M., Gerace, D., Sanvitto, D. \& Bajoni, D. Guided Bloch surface wave polaritons. Appl. Phys. Lett. 98, 121118 (2011).

30. Pirotta, S. et al. Strong coupling between excitons in organic semiconductors and Bloch surface waves. Appl. Phys. Lett. 104, 051111 (2014).

31. Lerario, G. et al. High-speed flow of interacting organic polaritons. Light Sci. Appl. 6, e16212 (2017)

32. Solnyshkov, D. D., Terças, H. \& Malpuech, G. Optical amplifier based on guided polaritons in GaN and ZnO. Appl. Phys. Lett. 105, 231102 (2014).

33. Walker, P. M. et al. Exciton polaritons in semiconductor waveguides. Appl. Phys. Lett. 102, 012109 (2013).

34. Rosenberg, I., Mazuz-Harpaz, Y., Rapaport, R., West, K. \& Pfeiffer, L. Electrically controlled mutual interactions of flying waveguide dipolaritons. Phys. Rev. B 93, 195151 (2016).

35. Ellenbogen, T. \& Crozier, K. B. Exciton-polariton emission from organic semiconductor optical waveguides. Phys. Rev. B 84, 161304 (2011).

36. Ciers, J. et al. Propagating polaritons in III-nitride slab waveguides. Phys. Rev. Appl. 7, 034019 (2017).

37. $\mathrm{Hu}, \mathrm{F}$. et al. Imaging exciton-polariton transport in $\mathrm{MoSe}_{2}$ waveguides. Nat. Photonics 11, 356-360 (2017).

38. Walker, P. M. et al. Ultra-low-power hybrid lightmatter solitons. Nat. Commun. 6, 8317 (2015).
39. Bahari, B. et al. Nonreciprocal lasing in topological cavities of arbitrary geometries. Science 358, 636-640 (2017).

40. Bandres, M. A. et al. Topological insulator laser: experiments. Science $\mathbf{3 5 9}$ eaar4005 (2018)

41. Zuniga-Perez, J. et al. Homoepitaxial nonpolar (10-10) ZnO/ZnMgO monolithic microcavities: towards reduced photonic disorder. Appl. Phys. Lett. 108, 251904 (2016).

42. Huang, M. H. et al. Room-temperature ultraviolet nanowire nanolasers. Science 292, 1897-1899 (2001).

43. Zamfirescu, M., Kavokin, A., Gil, B., Malpuech, G. \& Kaliteevski, M. ZnO as a material mostly adapted for the realization of room-temperature polariton lasers. Phys. Rev. B 65, 161205 (2002).

44. Chu, S., Olmedo, M., Yang, Z., Kong, J. Y. \& Liu, J. L. Electrically pumped ultraviolet ZnO diode lasers on Si. Appl. Phys. Lett. 93, 181106 (2008).

45. Vanmaekelbergh, D. \& Van Vugt, L. K. ZnO nanowire lasers. Nanoscale 3, 2783-2800 (2011)

46. Versteegh, M. A. M., Vanmaekelbergh, D. \& Dijkhuis, J. I. Room-temperature laser emission of ZnO nanowires explained by many-body theory. Phys. Rev. Lett. 108, 157402 (2012).

47. Levrat, J. et al. Condensation phase diagram of cavity polaritons in GaNbased microcavities: experiment and theory. Phys. Rev. B 81, 125305 (2010).

48. Jamadi, O. et al. Polariton condensation phase diagram in wide-bandgap planar microcavities: GaN versus ZnO. Phys. Rev. B 93, 115205 (2016).

49. Haug, H. \& Grob, K. Exciton laser theory. Phys. Lett. A 26, 41-42 (1967).

50. Schneider, C. et al. An electrically pumped polariton laser. Nature 497, 348-352 (2013). 